\title{
Bandwidth Analysis of Silicon PhC Filters for CWDM Systems Using FDTD-2D Method
}

\author{
Paulo de Tarso Neves, Jr. and Alexandre A. P. Pohl
}

\begin{abstract}
The Finite Difference Time Domain method is applied to the bandwidth calculation of a nanostructure known as PBG Air Bridge filter, which is formed by a semiconductor slab waveguide and an array of air holes. Its transmittance spectrum is calculated by the FFT of electromagnetic fields propagating in the structure. Results show that structures made of circular holes can be used in a CWDM grid between 1470 and $1610 \mathrm{~nm}$. It is shown that up to eight wavelengths may be inserted in this region, limited by the large bandwidth of such devices.
\end{abstract}

Index Terms-Photonic band gap (PBG), finite difference time domain method (FDTD), optical filters, nanostructures.

\section{INTRODUCTION}

$\mathrm{P}$ HOTONIC Crystal $(\mathrm{PhC})$ structures have turned to be the focus of many studies concerning their application in the optical communication area. PhCs are known to present frequency band gaps or stop bands, caused by a strong periodic variation of the refraction index, as seen by a wave along its propagation path. The determination of the guidance properties is achieved through the introduction of a defect in the structure, which is obtained by breaking the continuity of its periodic symmetry. Recently, PhCs have become the focus of research groups considering their application to WDM systems [1], particularly as optical filters for channel selection with sharp transmission resonance [2].

One structure of potential interest is a periodic array of circular holes filled by air in a strip of silicon (refraction index $n=3.4$ ), as shown in Fig. 1. Such microcavity can be realized by implementing a one-dimensional photonic crystal for confinement in one direction and employing index confinement in the remaining two directions. This work presents an analysis of such microcavity by means of the Finite-Difference Time-Domain technique (FDTD), showing how small changes in the periodic geometry and in the defect length affect the spectral filtering characteristic. Calculations show the influence on the center-frequency and width of the transmission spectrum due to changes in the defect length, hole

Manuscript received March 29, 2005. Revised May 4, 2006.

Paulo de Tarso Neves Jr. and Alexandre de Almeida Prado Pohl are with the Federal University of Technology - Paraná, Av. Sete de Setembro, 3165 , 80.230-901, Curitiba, Pr, Brazil. pohl@cpgei.cefetpr.br, Tel. +55-4133104695. The authors thank Coordenação de Aperfeiçoamento de Pessoal de Nível Superior (CAPES) and $\mathrm{CNPq}$ through the research grant $\mathrm{CNPq}$ 473.454/2003-3. size and distance between holes in the microcavity.

\section{METHODOLOGY}

\section{A. Strucuture Dimensions}

In this work the depth (in $z$ direction) of the structure is taking as infinitely large. This way, the wave propagation (in $x$ direction) can be calculated assuming the structure as a slab waveguide with a periodic pattern of circular air holes as shown in Fig. 1. The PhC is realized by implementing the onedimensional confinement in the $x$ direction, having an index confinement in the $y$ direction.

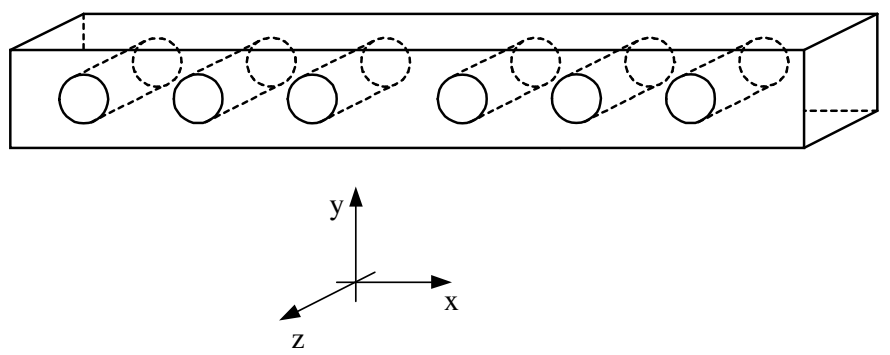

Fig. 1. One-dimension PhC structure. The wave propagation can be calculated assuming the structure as a slab waveguide, where the depth in $z$ direction in infinitely large.

In this work the FDTD-2D (Finite-Difference Time-Domain method in two dimensions) is used with a Perfectly Matched Layer (PML) absorbing boundary condition [3]. The microcavity is calculated in a square-cell grid having $\Delta x=\Delta y$ $=\Delta=\lambda / 200$ and a time-step $\Delta t=\Delta / \sqrt{2} c$, where $\lambda=1550$ $\mathrm{nm}$ and $c$ is the wave phase velocity in vacuum. Therefore, each cell has a $7.75 \mathrm{~nm} \times 7.75 \mathrm{~nm}$ size. The reason for choosing $\Delta$ so small is to guarantee the numerical stability, to avoid numerical dispersion in the algorithm [4] and to reduce the staircase effect, since the geometry of the holes is circular. The waveguide used in the calculations is $434 \mathrm{~nm}$ wide and is composed of an array of six circular air holes. For design purposes $R$ represents the hole radius, $P$ the center to center distance between the two consecutive holes and $D$ the center to center distance between the two innermost holes, which is termed here as the defect length. The radius of each hole is $R=$ $131.75 \mathrm{~nm}$ and their periodicity (center-to-center distance) is $P$ $=364.25 \mathrm{~nm}$. A $D=503.75 \mathrm{~nm}$ long defect is introduced in the center, as shown in Fig. 2. 


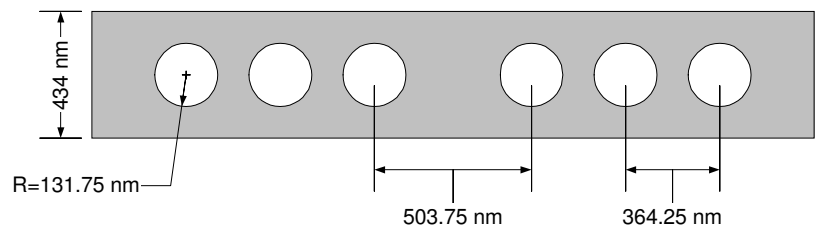

Fig. 2. Longitudinal cross-section view of the PhC. A $503.75 \mathrm{~nm}$ long defect is introduced in the center of a periodic array of holes.

These dimensions were chosen in order to implement the $\mathrm{PhC}$ waveguide and to ensure the resonant nature of the structure around the wavelength range $(1550 \mathrm{~nm})$ of interest for optical communication [2]. With such dimensions 800 cells in $\mathrm{x}$-axis are necessary for the main grid, resulting a length of $6.2 \mu \mathrm{m}$ for the numerical structure, as shown in Fig. 3.

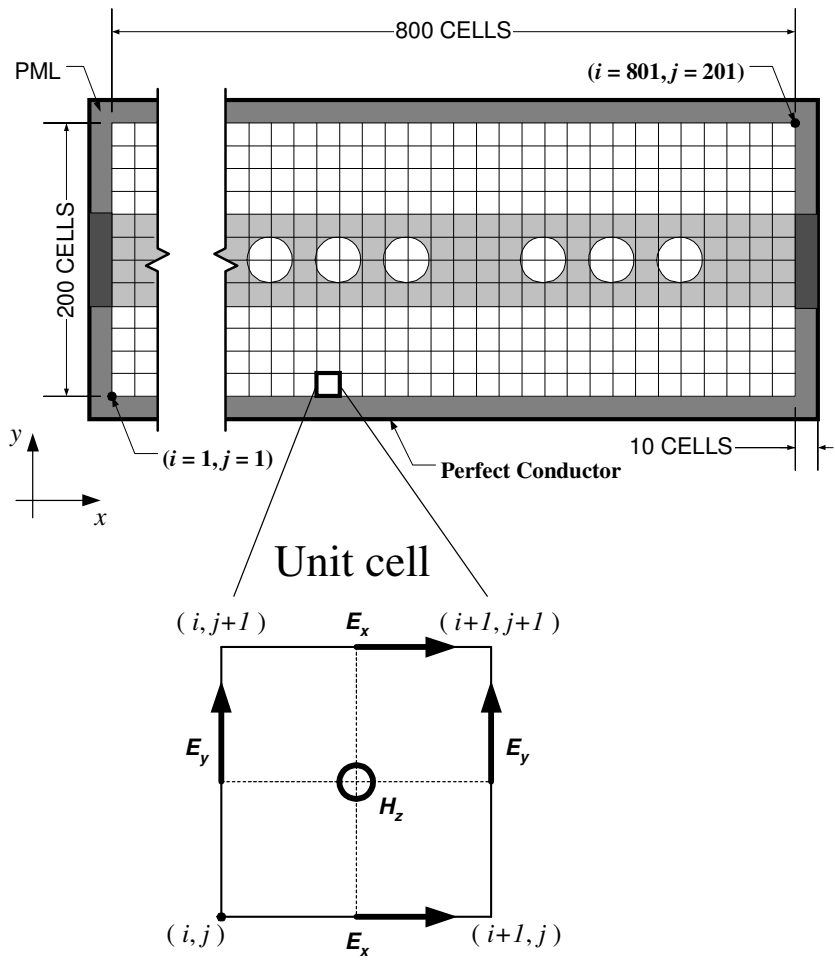

Fig. 3. Main grid and PML discretized by unit cells. The PML is around the main grid and a perfect conductor limits the computational domain.

The propagation analysis, using the FDTD notation, is focused on two dimensions $\mathrm{TE}_{\mathrm{z}}$ mode (transversal electric field concerning the $z$ axis) as there is no stopband in the $\mathrm{PhC}$ waveguide over the range of frequency used in optical communication (1550 nm) for the $\mathrm{TM}_{\mathrm{z}}$ mode [5].

\section{B. Temporal and Spatial Characteristic of the Input Source}

For the calculation of the electric and magnetic field strengths propagating in the slab waveguide a pulse waveform is launched at its input. Such a pulse is described by its time and spatial dependence as follows:

$$
H(y, t)=H_{z}(y) H_{t}(t)
$$

The time dependence is given by the expression:

$$
H_{t}(t)=H_{0} e^{-\left[\left(m-m_{0}\right) / m d e c\right]^{2}} \cos \left(2 \pi f_{0}\left(m-m_{0}\right) \Delta t\right)
$$

where $m$ is the time-step, $H_{0}=1, f_{0}=193.55 \mathrm{THz}, m_{0}=750$ and $m_{\text {decay }}=250$. The choice of $m_{\mathrm{o}}$ and $m_{\text {decay }}$ is done considering the range of frequencies comprised by the pulse in the region of interest. A source with such values represents a 9.134 fs Gaussian pulse modulating a $\lambda=1550 \mathrm{~nm}$ carrier, as shown in Fig. 4.

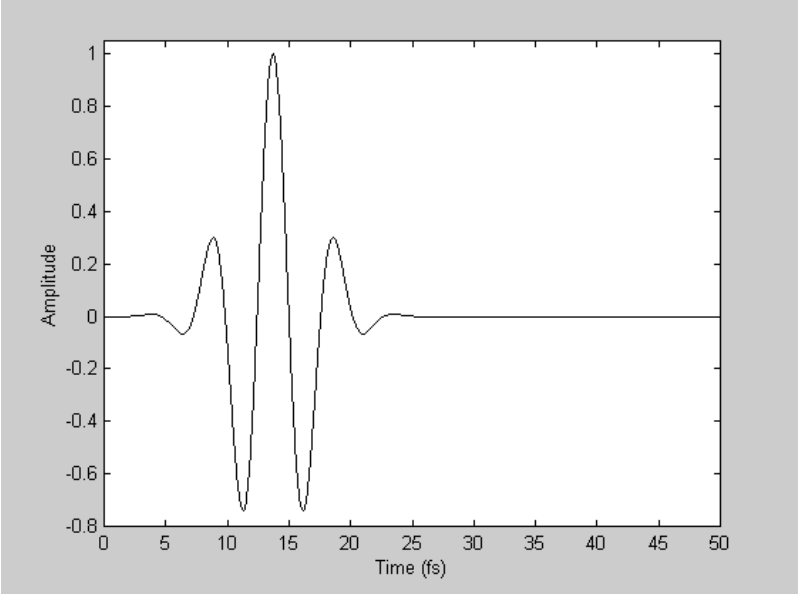

Fig. 4. Time behavior of the $H_{t}$ pulse waveform launched at the input of the slab waveguide.

The spectral intensity of the $H_{t}$ pulse waveform is shown in Fig. 5. One notes that a large amount of energy is concentrated around $f_{0}=193.55 \mathrm{THz}$. The source is placed at x-cell $i_{s}=20$ to avoid the localization of the input pulse at the PML interface.

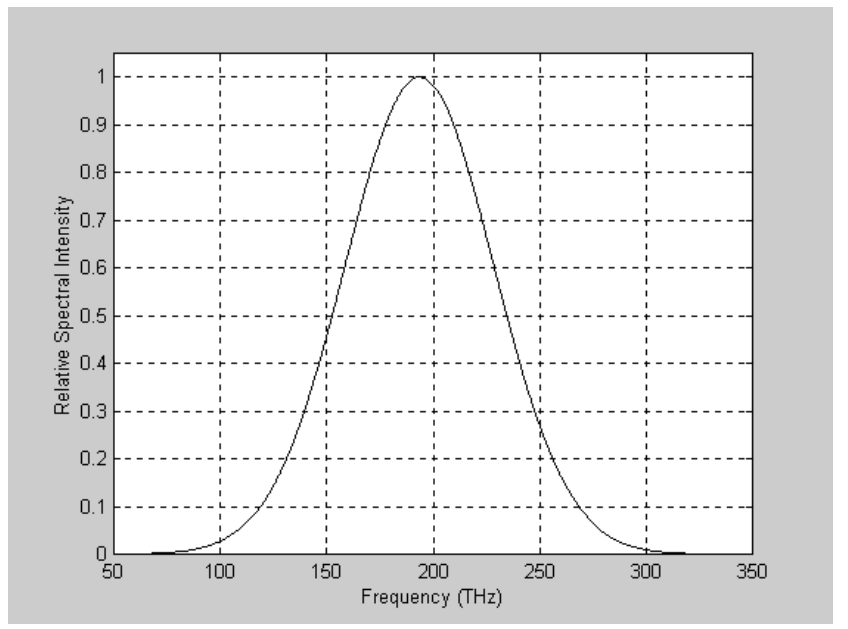

Fig. 5. Spectral intensity of the $H_{t}$ pulse waveform launched at the input of the slab waveguide. 
The pulse spatial behavior is calculated as the fundamental mode of a symmetric slab waveguide, whose width is $w=434$ $\mathrm{nm}$. Solving the characteristic equations, the propagation parameters for the slab waveguide (odd modes) are [6]:

$$
\begin{gathered}
\kappa=n_{1}^{2}\left(\frac{2 \pi}{\lambda_{0}}\right)^{2}-\beta^{2} \\
\gamma=\beta^{2}-n_{0}^{2}\left(\frac{2 \pi}{\lambda_{0}}\right)^{2} \\
\tan \kappa w=\frac{\gamma}{k}
\end{gathered}
$$

We have used the wavelength $\lambda_{0}=1550 \mathrm{~nm}$, the core refraction index $n_{1}=3.4$ (silicon) and the cladding refraction index $n_{0}=1$ (air), where $\beta$ is the mode propagation constant.

The $H_{z}$ component inside the slab is written as:

$$
H_{z 1}(y)=A \cos (\kappa y)
$$

for $|y| \leq w / 2$.

Outside the slab, the field $H_{z}$ must be evanescent. Therefore

$$
H_{z 2}(y)=A \cos (\kappa w / 2) e^{-\gamma(|y|-w / 2)}
$$

for $|y| \geq w / 2$.

The parameters $\kappa$ and $\gamma$ are linked to the propagation constant in the core and to the decay constant in the cladding of the slab waveguide. Fig. 6 shows the spatial intensity profile of the $H_{z}$ component as a function of the number of cells in the $\mathrm{y}$-axis.

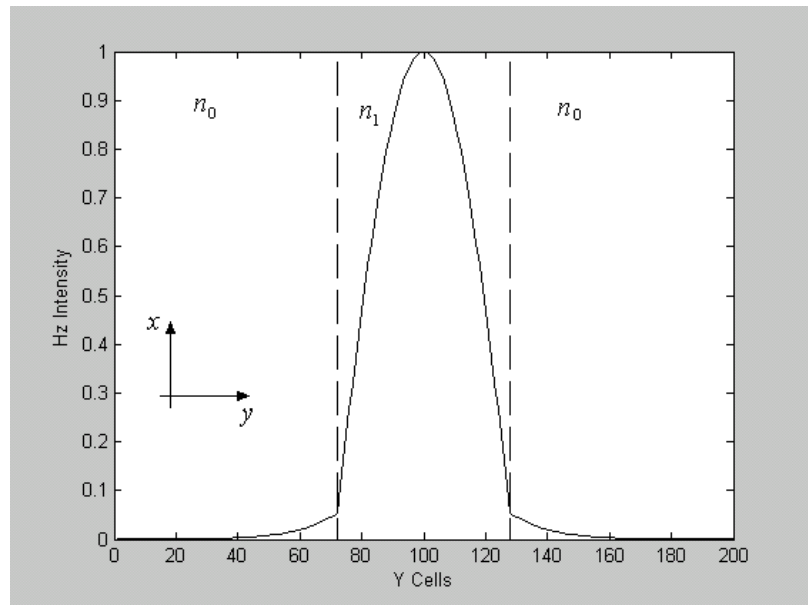

Fig. 6. Spatial distribution of the $H_{z}$ pulse waveform launched at the input of the slab waveguide. The center of the symmetric slab waveguide is at y-cell $j$ $=100$. Dashed lines represent the interface between the core (silicon) and the cladding (air) at y-cell $j=72$ and y-cell $j=128$, respectively.
The source can not remain active during the pulse propagation time through the structure, as reflections caused by the holes propagate back to the source and interfere with the launching field, modifying the input conditions. This way, the first hole is put far away from the source to avoid retroreflection. The center of the first hole is at x-cell $i=450$ (the source is at x-cell $i_{s}=20$ ) and after $\left(\mathrm{m}-m_{0}\right) / \mathrm{m}_{\text {decay }}=3$ the source is "turned-off". This is a simulation artifice and the real structure is a bit shorter. Since the output field intensity is normalized against the input, the fact that the source is turned off will not interfere on results.

\section{The Perfectly Matched Layer (PML)}

In order to simulate the extension of the grid to infinity Absorbing Boundary Conditions (ABCs) are implemented around the main grid, as shown in Fig. 3. The structure under analysis is modeled inside the main grid; fields calculated inside the main grid should not suffer significant interference from the boundary region. An ideal $\mathrm{ABC}$ allows waves to leave the main grid with minima reflections, avoiding interferences that could result in inaccurate field solutions.

In this work the split-field PML technique is used [3]. For the $\mathrm{TE}_{\mathrm{z}}$ mode, the $\mathrm{H}_{\mathrm{z}}$ spatial component is broken in two subcomponents, $\mathrm{H}_{\mathrm{zx}}$ and $\mathrm{H}_{\mathrm{zy}}$. Assuming a medium, called PML medium, with electric conductivity $\sigma$, magnetic loss $\sigma^{*}$, electrical permittivity $\varepsilon$ and magnetic permeability $\mu$, the propagation of a plane wave will be governed by a set of four equations:

$$
\begin{gathered}
\varepsilon \frac{\partial E_{x}}{\partial t}+\sigma_{y} E_{x}=\frac{\partial\left(H_{z x}+H_{z y}\right)}{\partial y} \\
\varepsilon \frac{\partial E_{y}}{\partial t}+\sigma_{x} E_{y}=\frac{\partial\left(H_{z x}+H_{z y}\right)}{\partial x} \\
\mu \frac{\partial H_{z x}}{\partial t}+\sigma_{x}^{*} H_{z x}=-\frac{\partial E_{y}}{\partial x} \\
\mu \frac{\partial H_{z y}}{\partial t}+\sigma_{y}^{*} H_{z y}=\frac{\partial E_{x}}{\partial y}
\end{gathered}
$$

For a theoretical perfect match (no reflection) between the PML medium and a lossless medium with electrical permittivity $\varepsilon$ and magnetic permeability $\mu$, the following conditions must be satisfied [3]:

For the interface normal to $\mathrm{x}$-axis

$$
\sigma_{y}=\sigma_{y}^{*}=0
$$




$$
\frac{\sigma_{x}}{\varepsilon}=\frac{\sigma_{x}^{*}}{\mu}
$$

For the interface normal to $y$-axis

$$
\begin{gathered}
\sigma_{x}=\sigma_{x}^{*}=0 \\
\frac{\sigma_{y}}{\varepsilon}=\frac{\sigma_{y}^{*}}{\mu}
\end{gathered}
$$

Thus, the PML medium can be characterized by the set of parameters $\left(\sigma_{x}, \sigma_{x}^{*}, \sigma_{y}, \sigma_{y}^{*}\right)$. Fig. 7 shows the right side of the computational domain where one can see the interfaces normal to $\mathrm{x}$-axis and its characteristic sets of parameters. Since silicon and air are considered in this work as lossless media, both have the set $(0,0,0,0)$.

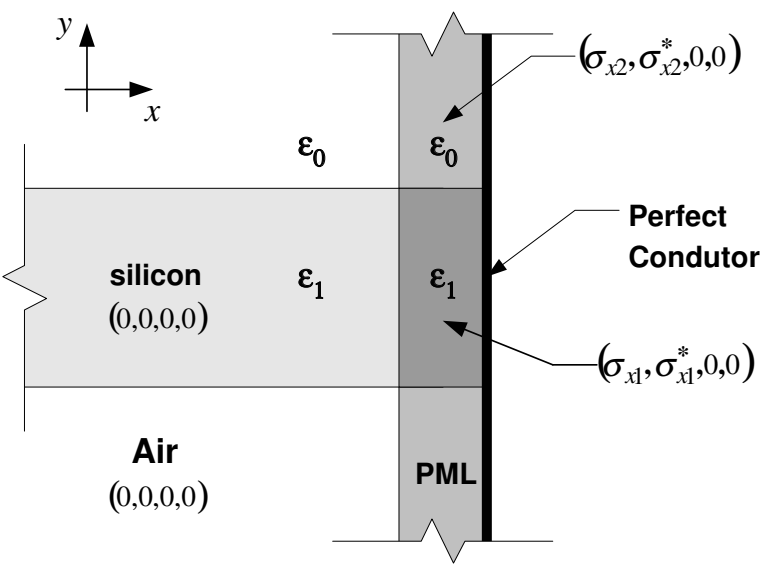

Fig. 7. Detail of the right side of the computational domain, where $\sigma_{x 1}$ and $\sigma_{x 2}$ are electric conductivities for the PML media matched with silicon and air respectively; $\sigma_{x 1}^{*}$ and $\sigma_{x 2}^{*}$ are magnetic losses for the PML media matched with silicon and air, respectively, and $\varepsilon_{0}$ and $\varepsilon_{1}$ are electrical permittivity for the vacuum and silicon, respectively.

The PML medium, as well as the main grid, needs to be of a limited thickness; thus, a perfect conductor is implemented around the PML layer (see Fig. 3). Therefore, the plane wave will cross the PML layer, will be reflected by the conductor and return to the main grid strongly attenuated due to PML losses. This reflection is called apparent reflection and should be as small as possible

For a PML medium with thickness $\delta$, an apparent reflection can be defined as [3]:

$$
R(\theta)=e^{-2 \eta \cos \theta \int_{0}^{\delta} \sigma(\rho) d \rho}
$$

where $\theta$ is the angle of the incident field with respect to the interface plane, $\eta$ is the impedance of the medium to be matched and $\sigma(\rho)$ is the electrical conductivity of the PML, as function of the distance $\rho$, with respect to the interface.

Abrupt variations of conductivity create numerical reflection. Thus, a smooth increase of $\sigma(\rho)$ must be forced. The gradual increase for $\sigma$ is achieved with the expression:

$$
\sigma(\rho)=\sigma_{m}\left(\frac{\rho}{\delta}\right)^{q}
$$

where $\sigma_{m}$ is the maximum value reached by $\sigma$ when $\rho=$ $\delta$. The parameter $q$ is known as the PML order.

The choice of $q$ for optimized absorption is done by considering the thickness $\delta$ and the maximum reflection desired for a specific angle. Choosing $R(0)$ as the maximum reflection for normal incidence, $\sigma_{m}$ will be:

$$
\sigma_{m}=\frac{(q+1)}{2 \eta \delta} \ln \left(\frac{1}{R(0)}\right)
$$

Numerical simulations $[7,8]$ demonstrated that an optimal choice for a broad range of applications using 10-cell-thick PML is $R(0) \approx e^{-16}$. Applying these values in (16) with unit square cells results:

$$
\sigma_{o p t} \approx \frac{(q+1)(16)}{(2 \eta)(10 \Delta)}=\frac{0,8(q+1)}{\eta \Delta}
$$

where $\Delta$ is the unit cell length.

In this paper the PML calculation is performed with $q=2$. Fig. 8 shows the gradual increase of $\sigma_{\text {opt }}$ on the right side of the PML (see Fig. 7) matched with the main grid. The difference in the $\sigma_{o p t}$ increase rate between the central (waveguide core) and the lateral (cladding) region is due to the electrical permittivity $\varepsilon$ being different in both parts.

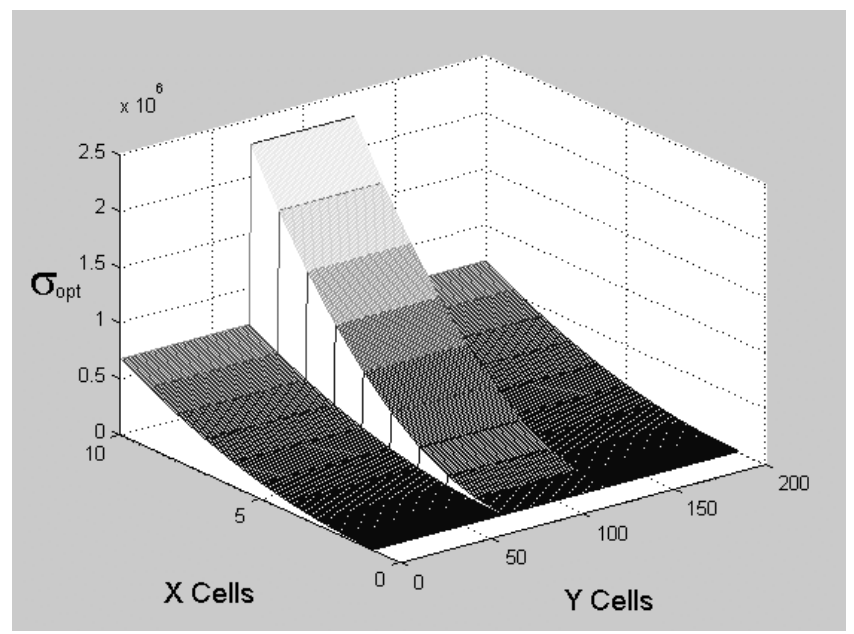

Fig. 8. Gradual increase of $\sigma_{\text {opt }}$ on the right side PML. The central prominent surface is the waveguide matched region. 


\section{RESULTS}

For the calculations, a code was written using the MATLAB language. Reflected and transmitted pulses were monitored at observation points before and after the holes. The Fast Fourier Transform (FFT) of these pulses, normalized to the spectrum of the input pulse, gives the reflection and transmission behavior in the structure. The number of time steps used in the calculation is $10^{5}$, which is enough to obtain spectra without loss of information. This maximum number of time steps is not optimized, but it is based on the examination of the resonance peak on the power spectrum. Since the resonance activity in the microcavity is related to the power in central frequency of the resonance peak, time step truncation before $4.10^{4}$ would lead to a significant reduction of the resonance peak and results would show smaller transmission. Simulations were done using a PC with $2.6 \mathrm{GHz}$ clock and 1Gbyte RAM. For $10^{5}$ time steps the total calculation time is about 5 hours.

Fig. 9 shows the transmission spectrum of the $\mathrm{PhC}$ waveguide showing the stopband. In this case, the central distance between the holes is $364.25 \mathrm{~nm}$. The stopband is seen along the range of wavelengths used in optical communication (1270nm to $1610 \mathrm{~nm})$.

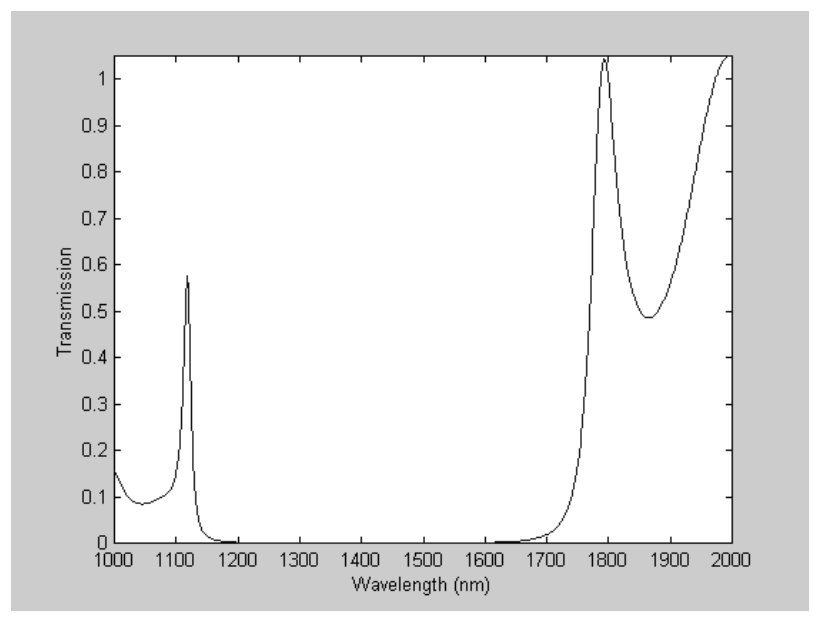

Fig. 9. Transmission spectrum of the $\mathrm{PhC}$ waveguide, where the central distance between the holes is $364.25 \mathrm{~nm}$.

The introduction of a defect generates a transmission peak in the stopband region, as shown in Fig. 10. In this case, the defect length is $D=503.75 \mathrm{~nm}$ and there is a transmission peak located at $1463.8 \mathrm{~nm}$, whose maximum value is $T_{\max }=0.794$. Such result shows that the structure presents a resonant behavior and can be used as an optical filter.

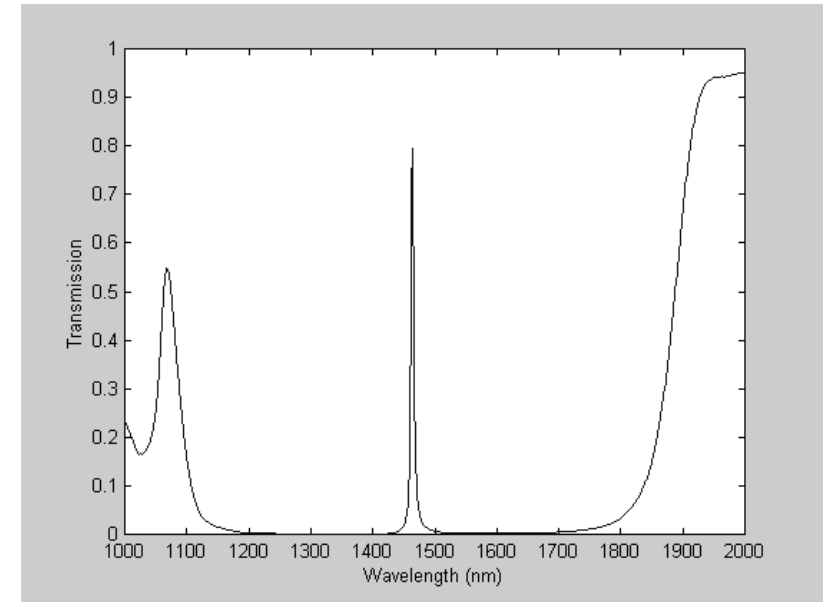

Fig. 10. Transmission spectrum of the $\mathrm{PhC}$ waveguide, showing the resonant peak at $1463.8 \mathrm{~nm}$, when the defect length is $503.75 \mathrm{~nm}$.

The time response of the field $H_{z}$ intensity at observation points, before the first hole (input) and after the last hole (output) is showed in Fig. 11. One observes oscillation at the output field during a long time after the total input pulse has faded away, which attests the resonant nature of the structure.

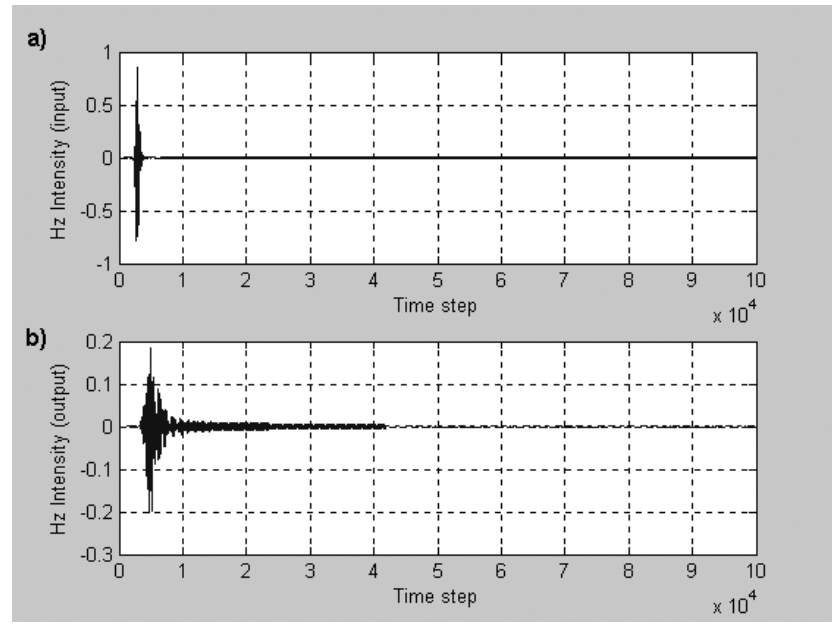

Fig. 11. a) $\mathrm{Hz}$ intensity at observation point $(i, j)=(400,100)$, before the first hole (input). b) $H z$ intensity at observation point $(i, j)=(750,100)$, after the last hole (output).

Fig. 12 (a) shows the field $H_{z}$ between the time-step 30000 and 32500 at the output; the $H_{z}$ behavior follows a dumped sine wave. Fig. 12 (b) shows a cycle of such wave between the time-step $m_{i}=30220$ and $m_{f}=30487$. This cycle has a wavelength $\lambda=c\left(m_{f}-m_{i}\right) \Delta t=1463.2 \mathrm{~nm}$, where $c$ is the vacuum wave phase velocity and $\Delta \mathrm{t}$ is the simulation time increment; this value is close to the central wavelength of the transmission peak shown in Fig. 10. 


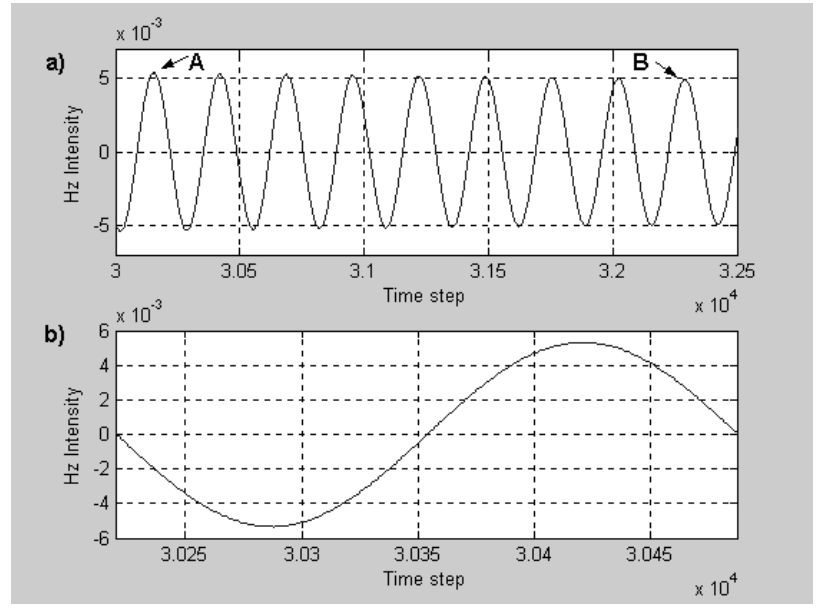

Fig. 12. a) $H_{z}$ intensity at output between time steps $n_{i}=30000$ a $n_{f}=$ 32500 , note the fading comparing points $\mathrm{A}$ and B. b) Wave cycle between time steps $n=30220$ a $n=30487$.

The electromagnetic resonant microcavity created by inserting the defect in the $\mathrm{PhC}$ structure, like other similar cavities found in lasers and filters, is characterized by two main quantities: the modal volume $\mathrm{V}$ and the quality factor $\mathrm{Q}$. The modal volume is associated with the volume occupied by the electromagnetic radiation in the cavity and the quality factor indicates its ability in selecting a certain frequency. High Q's and small V's are highly desirable due to their impact in the high finesse required for lasers and filter applications. Generally, one focus on the $\mathrm{Q} / \mathrm{V}$ relationship associated with the cavity. For $\mathrm{PhC}$ structures the fine tuning of the defect length, the period or the hole diameter modifies the cavity parameters, changing its $\mathrm{Q} / \mathrm{V}$ relationship.

As will be shown in the next sections, widening the defect length has the effect of increasing the resonant wavelength. On the same token, changing the spacing between the holes or the hole diameter will also cause the resonant wavelength to change. The wavelength shift has its origin in the displacement of the PhC stopband as the structure dimensions are changed. By wisely tuning the microcavity dimensions special filter characteristics can be realized.

\section{A. Changing the Defect Length}

Setting $D_{\text {ref }}=503.75 \mathrm{~nm}$ as a reference, the length of the defect is varied using steps of $\Delta=7.75 \mathrm{~nm}$. Fig. 13 shows the superposition of spectra varying the defect length from $D=$ $496 \mathrm{~nm}$ to $D=565.75 \mathrm{~nm}$. Defect lengths smaller than $496 \mathrm{~nm}$ results in maximum transmission peaks, $T_{\max }$, lower than 0.7, which are not shown in the Fig. 13.

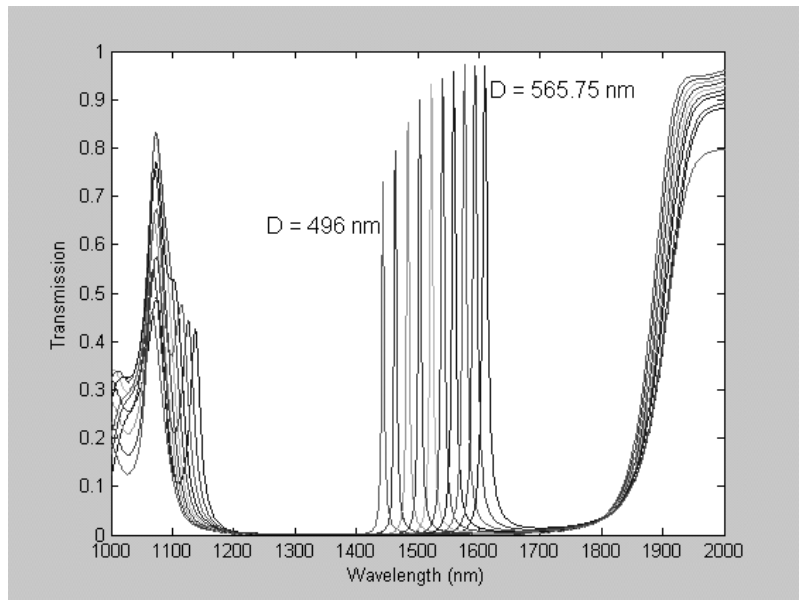

Fig. 13. Superposition of spectra varying the defect length from $D=496 \mathrm{~nm}$ to $D=565.75 \mathrm{~nm}$.

One sees that the resonant wavelength changes are motivated by a slight change in the $\mathrm{PhC}$ stopband region. Moreover, the intensity of the transmission peaks also grows as the wavelength increases. This behavior is explained by scattering losses caused by the holes. For instance, when the defect length is small, the holes are closer to each other and more radiation in the cavity is scattered by them. As a consequence, the intensity of the transmission peak sees a reduction. As the defect length increases, the holes move apart and the propagating mode sees less scattering. This way, the intensity of the transmission peak increases.

It is also important to remember that the FDTD calculations in this work are made in 2 dimensions. Scattering fields in the $z$ direction are not computed. This fact leads to an apparent peak intensity that may be greater than it really is, as scattering losses certainly occur in that direction.

The bandwidth of these transmission peaks is also calculated (see Fig. 14) as a function of the wavelength of the maximum transmission peak for each defect length. Defect lengths longer than $565.75 \mathrm{~nm}$ result in bandwidths greater than $10 \mathrm{~nm}$. The same figure shows also the behavior of the cavity quality factor. One observes that the quality factor decreases for increasing wavelengths. If one thinks the $\mathrm{PhC}$ structure as a Fabry-Perot etalon, where holes on each side play the role of mirrors, the decreasing of $\mathrm{Q}$ is equivalent to the detuning of the etalon.

The physical mechanism behind the bandwidth increase is not so clear at this point. We believe that field phase mismatch at the hole walls is responsible for this occurrence. Again, the comparison with the etalon may be helpful. It is well known that the quality of the mirrors is responsible for the finesse of the etalon. Extending the analogy to the $\mathrm{PhC}$ structure, the phase matching conditions of the incident and scattered electromagnetic fields at the hole walls lead to the modification of the bandwidth. This phenomenon has already been experimentally observed in similar structures, although no explanation was given for this behavior [9]. 


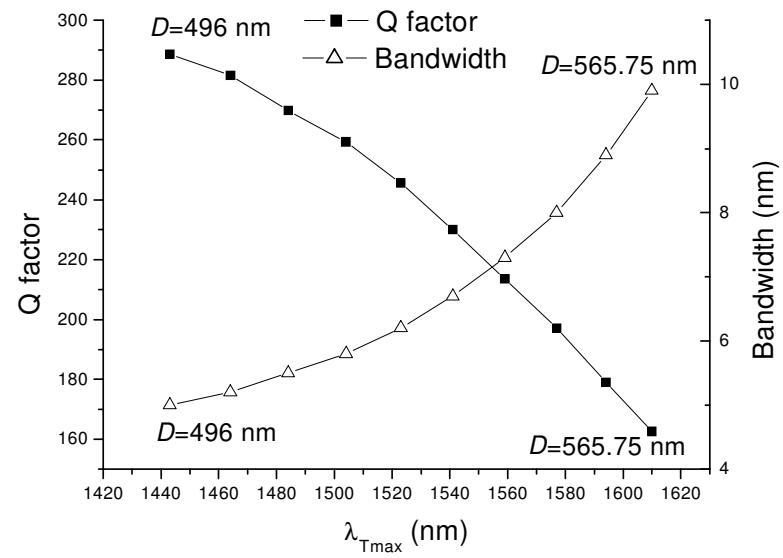

Fig. 14. Filter bandwidth and Q factor as a function of wavelength of the transmission peak for each defect length.

From the estimated bandwidths it is possible to find applications for these filters. Their application, for instance, in densely channel packed WDM systems would be of no value, since such systems require channel spacing as narrow as 0.1 $\mathrm{nm}$ [10]. However, their use in the so-called CWDM (Coarse WDM) systems, where filter bandwidth requirements are more relaxed, might be a possibility as the recommendation for channel spacing considers a wavelength separation of $20 \mathrm{~nm}$ in the range from $\lambda=1270 \mathrm{~nm}$ to $\lambda=1610 \mathrm{~nm}$ [11].

Looking closer at this application, a simulation effort is done to place as many channels as possible in such range. By varying the defect length with steps smaller than $7.75 \mathrm{~nm}$, while maximizing the transmission peak $\left(T_{\max }>0.8\right)$, eight channels can be centered in that interval. Fig. 15 shows the simulation results.

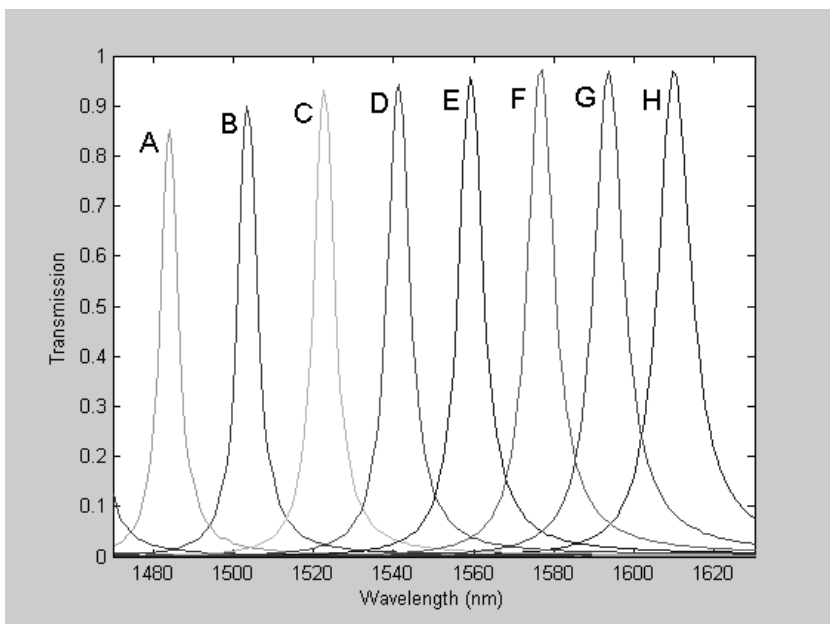

Fig. 15. Detail of Fig.13 showing eight channels between $1470 \mathrm{~nm}$ and 1630 $\mathrm{nm}$. The last channel (H) presents $\lambda_{\text {Tmax }}=1610 \mathrm{~nm}$.

Fig. 16 presents the necessary defect length associated with the maximum transmission peak of each channel shown in Fig. 15.

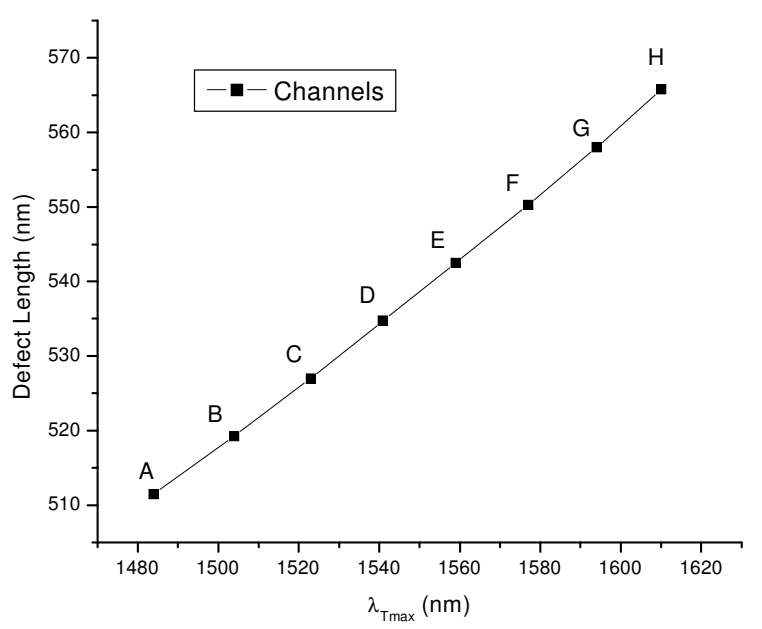

Fig. 16. Central wavelength $\lambda_{\text {Tmax }}$ for each defect length between $1470 \mathrm{~nm}$ and $1610 \mathrm{~nm}$.

\section{B. Changing the Radius}

Further calculations were done to verify the spectrum transmission behavior by changing the radius of the holes in the $\mathrm{PhC}$ structure, while maintaining $P$ and $D$ fixed at $364.25 \mathrm{~nm}$ and $503.75 \mathrm{~nm}$, respectively. Setting $R_{\text {ref }}=$ $131.75 \mathrm{~nm}$ as the reference, the radius of holes can be varied maintaining a step $\Delta=7.75 \mathrm{~nm}$.

Fig. 17 shows the behavior of the filter bandwidth as function of the transmission peak wavelength, by varying the radius from $R=108.5 \mathrm{~nm}$ to $R=139.5 \mathrm{~nm}$. Radii smaller than $108.5 \mathrm{~nm}$ result in bandwidths greater than $12 \mathrm{~nm}$, radii greater than $139.5 \mathrm{~nm}$ result $T_{\max }$ smaller than 0.7. Due to the increasing bandwidth for radius smaller than $108.5 \mathrm{~nm}$, it is not possible to lodge more than 5 CWDM channels, with transmission peak greater than 0.7 and bandwidth smaller than $10 \mathrm{~nm}$ in the region of interest between $1470 \mathrm{~nm}$ and $1610 \mathrm{~nm}$.

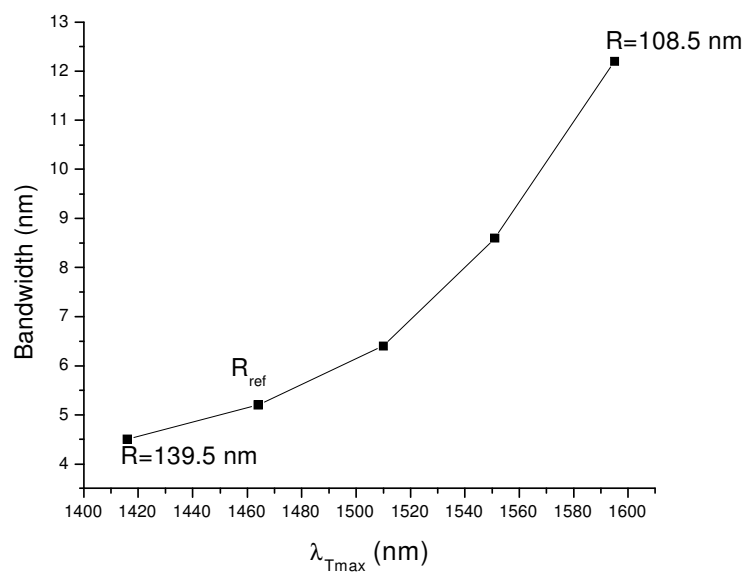

Fig. 17. Filter bandwidth as function of wavelength of the transmission peak for each radius. 


\section{Changing the Period}

By changing the period of the holes in the PhC structure, while maintaining $D$ and $R$ fixed at $503.75 \mathrm{~nm}$ and $131.75 \mathrm{~nm}$, respectively; a variation of the filter bandwidth is also observed. Setting $P_{\text {ref }}=364.25 \mathrm{~nm}$ as the reference, the period of holes is varied in steps of $\Delta=7.75 \mathrm{~nm}$. Fig. 18 shows the behavior of the filter bandwidth as a function of the transmission peak wavelengths for periods starting at $P=$ $325.5 \mathrm{~nm}$ and ending at $P=418.5 \mathrm{~nm}$. One sees that the bandwidth is minimum between $P=364.25 \mathrm{~nm}$ and $P=$ $379.75 \mathrm{~nm}$. Periods greater than $418.5 \mathrm{~nm}$ result in values of $T_{\max }<0.7$.

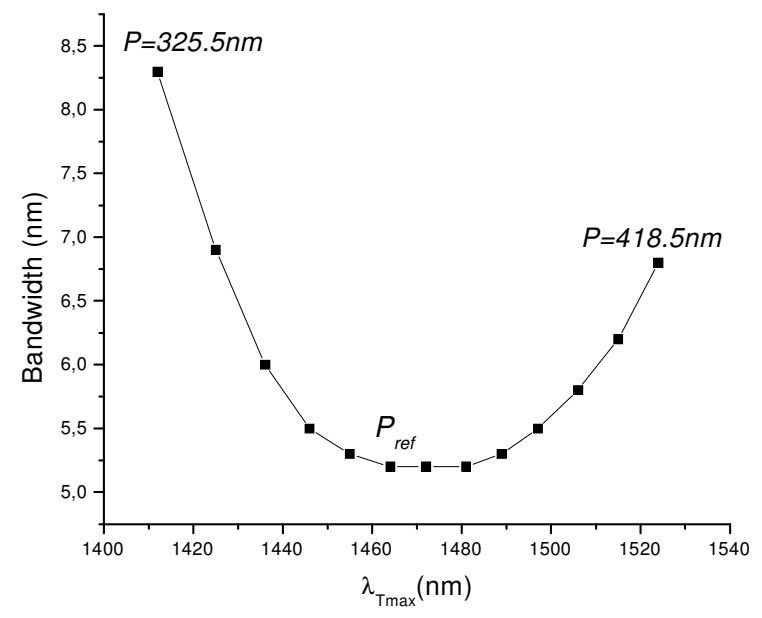

Fig. 18. Filter bandwidth as function of wavelength of the transmission peak for each period.

Again, no results are found in order to lodge $8 \mathrm{CWDM}$ channels, with transmission peak greater than 0.7 and bandwidth smaller than $10 \mathrm{~nm}$, between $1470 \mathrm{~nm}$ and 1610 $\mathrm{nm}$.

It is seen from Fig. 18 that the bandwidth reaches a minimum at $1470 \mathrm{~nm}$ and starts rising again. Although not observed when the defect length and the hole radius were altered in the same wavelength range, this behavior helps to confirm the character of the $\mathrm{PhC}$ structure as an etalon, as periodic changes reproduce the phase conditions that lead to the same bandwidth.

\section{Group Delay and Dispersion}

Another important characteristic of filter devices used in optical communication concerns the group delay parameter. The transmitted light group delay is calculated by the expression [12]

$$
\tau_{T}=-\frac{\lambda^{2}}{2 \pi c} \frac{\partial \theta_{T}}{\partial \lambda}
$$

where $\lambda$ is the wavelength in vacuum, $c$ is the wave phase velocity in vacuum and $\theta_{T}$ is the transmission phase.

However, the dispersion parameter $D_{T}$, that is, the group delay variation rate with respect to the wavelength (expressed in $\mathrm{ps} / \mathrm{nm}$ ) is given by

$$
D_{T}=\frac{\partial \tau_{T}}{\partial \lambda}=-\frac{2 \pi c}{\lambda^{2}} \frac{\partial^{2} \theta_{T}}{\partial \omega^{2}}
$$

Using this definition, the dispersion caused by the structure with the dimensions presented in Fig. 2 is calculated and the result is seen in Fig.19-b. One observes the occurrence of maximum and minimum values of dispersion at the transmission peak region. Dispersion values $\left(D_{T}\right)$, calculated in $\mathrm{ps} / \mathrm{nm}$ and around $\lambda_{\mathrm{Tmax}}$, for structures considered in this work are situated in the range $-0.06<D_{T}<0.06$.

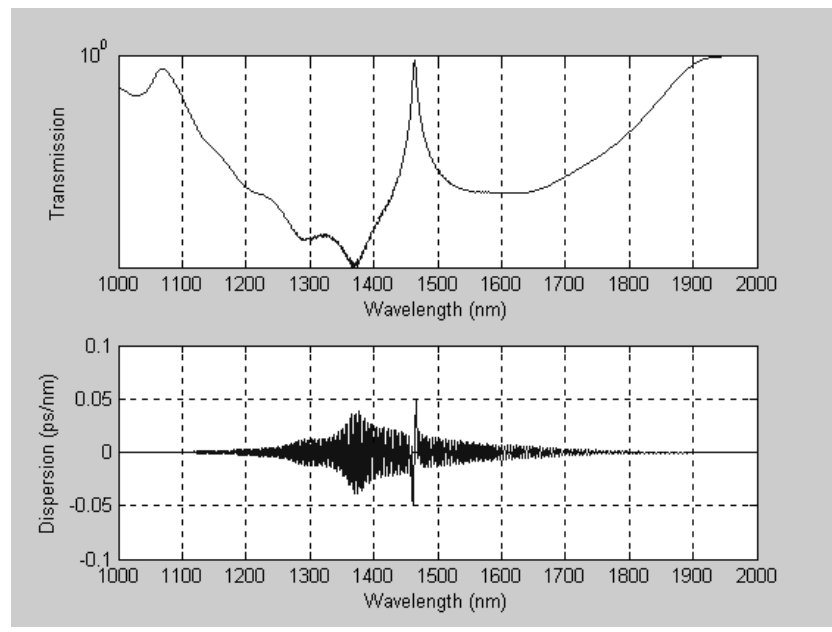

Fig. 19. a) Fig. 10 in $\log$ scale. b) Dispersion as a function of $\lambda$.

Fig. 20 shows a comparison of the dispersion and bandwidth behavior as a function of $\lambda_{\text {Tmax }}$ by changing the period of the $\mathrm{PhC}$ structure. One sees that when the bandwidth is minimum, the dispersion is maximum.

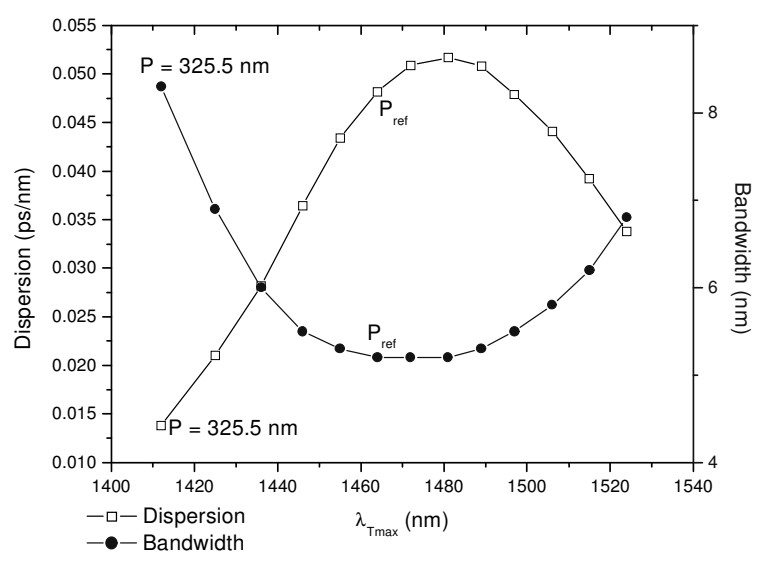

Fig. 20. Dispersion and bandwidth as a function of $\lambda_{\text {Tmax }}$. 


\section{CONCLUSION}

The spectrum analysis of $\mathrm{PhC}$ structures is performed using the FDTD-2D method concerning the transmission spectra in the stopband region and the bandwidth of each resonance peak. Results show that the geometry and the propagation mode determine the presence of the stopband in a region of spectrum. By changing the length of the defect, the radius of the holes or the distance between the holes, the location of the resonant modes within the stopband can be customized. No combination of geometrical parameters was found in order to result a bandwidth smaller than $5 \mathrm{~nm}$ with a transmission value greater than 0.7 for the resonance peaks in the region between $1470 \mathrm{~nm}$ and $1610 \mathrm{~nm}$. Recently, experimental results for a similar structure [13] have indeed shown a $7 \mathrm{~nm}$ FWHM, which is in good agreement with the results of this work. Due to this fact, the structures could be potentially applied as optical filters in Coarse Wavelength Multiplexing Systems (CWDM) [14], where filter bandwidth requirements are more relaxed, and wavelength spacing is $20 \mathrm{~nm}$. A limit for the maximum number of wavelengths in the 1470-1610 nm range is set by the defect length of the device, which shows bandwidths greater than $10 \mathrm{~nm}$ for $D>565.75 \mathrm{~nm}$.

Results show that small changes in geometry will cause a significant change in the transmission spectrum. This imposes stringent demands on the nanoscale-dimensional control of the fabrication process in order to achieve reproducibility. New techniques such as electron beam (e-beam) lithography [15] or deep-UV (DUV) lithography [16] are strong candidates for constructing such $\mathrm{PhC}$ structures.

Several experimental works $[9,13]$ have already demonstrated the feasibility of fabricating these $\mathrm{PhC}$ filters and have shown that the fine tuning of the cavity parameters can drastically change its transmission properties. However, an improvement of the theoretical optimization techniques is still demanding, as the concepts behind the understanding of the physical mechanisms are not yet mature, as pointed out by a recent study on the problem [17].

\section{REFERENCES}

[1] A. D'Orazio, M. De Sario, V. Petruzzelli and F. Prudenzano, "Photonic band gap filter for wavelength division multiplexer," Optics Express, Vol. 11, No. 3, pp. 230-239, 2003.

[2] J. C. Chen, H. A. Haus, S. Fan., P. R. Villeneuve and J. D. Joannopoulos, "Optical filters from photonic band gap air bridges," $J$. Lightwave Technology, Vol. 14, pp. 2575-2019, 1996.

[3] J. -P. Berenger, "A perfectly matched layer for the absorption of electromagnetic waves," J. Computat. Phys., Vol. 114, pp. 185-200, 1994.

[4] A. Taflove, and S. C. Hagness, Computational Electrodynamics, Artech House, $2^{\text {nd }}$ edition., 2000.

[5] P. T. Neves Jr., Aplicação do método FDTD na análise de filtros ópticos em cristais fotônicos, M. S. thesis, CPGEI, Centro Federal de Educação Tecnológica do Paraná, 2004.
[6] A. H. Cherin, An introduction to optical fibers, McGraw-Hill, 1983

[7] S. D. Gedney, "An anisotropic perfectly matched layer absorbing media for the truncation of FDTD lattices", IEEE Trans. Antennas and Propagation, Vol. 44, pp. 1630-1639, 1996.

[8] L. He, FDTD - Advances in sub-sampling methods, UPML, and higherorder boundary conditions, M. S. thesis, University of Kentucky, 1997.

[9] K.Y. Lim et al, "Photonic Band-Gap Waveguide Microcavities: Monorails and Air Bridges", J. Vac. Sci. Technol. B 17(3), pg 11711174, 1999.

[10] ITU-T, G.694.1, "Spectral grids for WDM applications: DWDM frequency grid", 06/2002.

[11] ITU-T, G.694.2, "Spectral grids for WDM applications: CWDM frequency grid", 06/2002.

[12] T. Erdogan, "Fiber grating spectra," J. Lightwave Technology, Vol. 15, pp.1277-1294, 1997.

[13] A. S. Jugessur, P. Pottier and R. M. De La Rue: "Engineering the filter response of photonic crystal microcavity filters," Optics Express, Vol. 7 , No. 12, pp. 1304-1312, 2004.

[14] P. T. Neves Jr., and A. A. P. Pohl, "PBG optical filters for CWDM systems," Proc. 4th IASTED-Multiconf. Wireless and Optical Communication, Banff, Canada, July 2004.

[15] M. Loncar, D. Nedeljkovic', T. Doll, J. Vuckovic', A. Scherer, and T. P. Pearsall, "Waveguiding in planar photonic crystals," Apll. Phys. Lett., Vol. 77, pp. 1937-1939, 2000.

[16] M. Settle, M. Salib, A. Michaeli and T. F. Krauss, "Low loss silicon on insulator photonic crystal waveguides made by $193 \mathrm{~nm}$ optical lithography," Optics Express, Vol. 14, No. 6, pp. 2440-2445, 2006.

[17] P. Lalanne, S. Mias, and J.P. Hugonin, "Two Physical Mechanisms for Boosting the Quality Factor to Cavity Volume Ratio of Photonic Crystal Microcavities", Optics Express, vol. 12, no. 3, pp. 458-467, 2004.

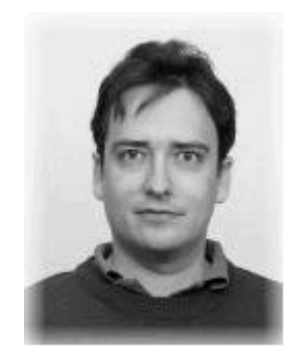

Paulo de Tarso Neves Junior was born in Cachoeiro de Itapemirim, Brazil, in 1973. He received the Bachelor degree in electrical engineering from the Federal University of Espirito Santo (UFES), Brazil, in 1998, and the Master degree in electrical engineering from the Federal Center of Technological Education of Parana (CEFET-PR), Brazil, in 2004. He is currently a PhD student at UTFPR. His research interests include photonic crystals, numerical methods applied to wave propagation and fast tuning in Fiber Bragg Gratings.

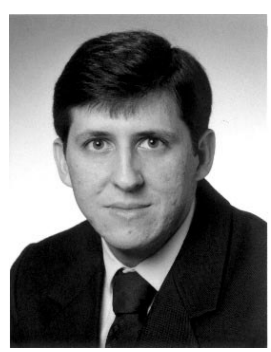

Alexandre de Almeida Prado Pohl received the B.S. and M.S. degree in Physics in 1983 and 1987, respectively, from the State University of Campinas, Brazil and the Ph.D. degree in electrical engineering in 1994 from the Technical University of Braunschweig, Germany. From 1995 to 2000 he worked as an application engineer and area coordinator at the Furukawa, Inc branch in Brazil. Since 2001 he has been with the electrical engineering department of the Federal University of Technology, Curitiba, Brazil, where he leads a research group working with optical fiber communications and photonic devices. He is a member of the Optical Society of America (OSA), the Brazilian Telecommunications Society (SBrT) and the Brazilian Microwave and Optoelectronics Society (SBMO). 